\title{
Development of a novel histologic diagnostic algorithm for hepatic graft-versus-host disease
}

\author{
Ashley E Stueck ${ }^{1}$, Thomas D Schiano ${ }^{2}$ and M Isabel Fiel ${ }^{3}$ \\ ${ }^{1}$ Dalhousie University, Department of Pathology, Halifax, NS, Canada; ${ }^{2}$ Icahn School of Medicine at Mount \\ Sinai, Division of Liver Diseases, New York, NY, USA and ${ }^{3}$ Icahn School of Medicine at Mount Sinai, \\ Department of Pathology, New York, NY, USA
}

\begin{abstract}
The histopathologic diagnosis of hepatic graft-versus-host disease post bone marrow and stem cell transplantation can be challenging, but timely and unambiguous diagnosis is essential for appropriate patient management. To address this diagnostic dilemma, we identified histologic features specific for hepatic graftversus-host disease and developed a diagnostic algorithm. Two hepatopathologists blindly evaluated 40 liver biopsies from patients with clinically and biologically confirmed graft-versus-host disease, as well as $\mathbf{4 4}$ controls, for percent bile duct loss, bile duct damage, intraepithelial lymphocytes, ductular reaction, acidophilic bodies/10 high power fields (HPF), cholestasis, portal and lobular inflammation, and endotheliitis. Compared with controls, graft-versus-host disease cases had significantly more bile duct loss $(P<0.0001)$, bile duct damage $(P=0.0002)$, cholestasis $(P<0.0001)$, and acidophilic bodies/10 HPF $(P=0.0006)$, as well as significantly less ductular reaction $(P<0.0001)$. Significance was maintained with a drug-induced liver injury-only control group. No histologic differences were noted in acute versus chronic graft-versus-host disease, nor cholestatic versus hepatitic types. An algorithm to predict likelihood of graft-versus-host disease was developed, with a three-tiered scoring system: 1-2 not, 3-4 probable, and 5-8 unequivocal graft-versus-host disease. This algorithm had a sensitivity of $93 \%$, specificity of $93 \%$, and accuracy of $92 \%$. We identified histologic features with specificity for hepatic graft-versus-host disease and developed a simple algorithm for pathologists to predict its likelihood, distinguishing this critical diagnosis promptly from mimickers having vastly different treatments and prognoses. Modern Pathology (2018) 31, 442-451; doi:10.1038/modpathol.2017.151; published online 1 December 2017
\end{abstract}

Hepatic graft-versus-host disease occurs in patients post allogeneic stem cell or bone marrow transplant, and rarely in other situations, such as post solid organ transplantation or after blood transfusion. Over $80 \%$ of these patients develop liver abnormalities, commonly elevated liver chemistry tests, and over $40 \%$ develop hepatic graft-versus-host disease. $^{1,2}$ The etiology of the liver abnormalities may be related to the stem cell or bone marrow transplant, such as hepatic graft-versus-host disease or recurrent disease, its associated therapies, such as drug-induced liver injury, infections, and total parenteral nutrition-related injury, or owing to unrelated intrinsic liver disease..$^{2,3}$ In particular, drug-induced liver injury is a very challenging diagnosis in this setting and understandably so because these patients are receiving multiple drug

Correspondence: Dr AE Stueck, MD, FRCPC, Dalhousie University/Nova Scotia Health Authority 715-5788 University Avenue, Halifax, NS B3H 2Y9, Canada.

E-mail: Ashley.stueck@dal.ca

Received 8 July 2017; revised 21 September 2017; accepted 22 September 2017; published online 1 December 2017 regimens, many of which have potential hepatotoxicity. Furthermore, many of these entities in the differential diagnosis have similar clinical and biochemical presentations. As treatments and prognoses are vastly different, diagnosis of hepatic graftversus-host disease is critical. Several hepatic graftversus-host disease prediction scores have been developed; the National Institutes of Health Scoring System is common in clinical practice, and incorporates serum values of total bilirubin, alanine aminotransferase, and alkaline phosphatase. ${ }^{4,5}$ However, recent studies have shown that this system has sensitivity of only $55 \%$ and specificity of $53 \% .6,7$ Thus, current non- and minimally invasive tests, and the probability scores based upon them, often fail to reliably distinguish hepatic graft-versus-host disease from its mimickers.

The pathologic diagnosis of hepatic graft-versushost disease is challenging, and most previous studies lacked control groups. ${ }^{8-17}$ Few studies with robust blinded methodology and control groups exist, and include Shulman et al., ${ }^{18}$ who identified an extensive list of histologic features seen more in hepatic graft-versus-host disease patients compared 
with a control group, including acidophilic bodies, bile duct loss, intraepithelial lymphocytes, and absence of portal inflammation. Snover et al. ${ }^{19}$ described endotheliitis as being present more frequently in hepatic graft-versus-host disease compared with controls. Several groups have published consensus guidelines on the histologic features of hepatic graft-versus-host disease, and recommendations for diagnosis and terminology, often in three to four-tiered scoring systems. ${ }^{4,20,21}$ A study with a strong control group, which includes the most common clinical and histologic mimickers of hepatic graft-versus-host disease, such as drug-induced liver injury, is needed. Furthermore, simplification of this challenging diagnosis for pathologists, such as by combining significant histologic features into an algorithmic tool, would be of great utility.

The objective of the current study was, first, to identify any histologic features that may be more specific to hepatic graft-versus-host disease as compared with a control group, which specifically included cholestatic drug-induced liver injury and vanishing bile duct syndrome pattern of druginduced liver injury. In addition, we sought to determine whether histologic differences exist between cholestatic and hepatitic clinical presentations of hepatic graft-versus-host disease. Finally, our goal was to develop a histologic-based algorithm for diagnosis of hepatic graft-versus-host disease using the identified histologic parameters.

\section{Materials and methods}

Forty liver biopsies from adult patients post allogeneic stem cell or bone marrow transplant with clinically confirmed hepatic graft-versus-host disease and absence of other intrinsic liver disease were examined, along with 44 control biopsies. The control etiologies included nine hepatitic druginduced liver injury, nine steatohepatitis, eight cholestatic drug-induced liver injury, seven normal livers, six cholestatic total parenteral nutritionrelated injury, and five vanishing bile duct syndrome pattern of drug-induced liver injury. Two of the cholestatic drug-induced liver injury controls had a cholestatic hepatitis histologic pattern. The druginduced liver injury control cases had causality assessed by expert hepatologists, with identification of etiologic agent(s), and exclusion of other liver injuries with clinical, serologic, and pathologic testing.

Cases were selected via search of the laboratory information system of the Department of Pathology at The Mount Sinai Hospital using specific search terms such as 'graft-versus-host disease', 'druginduced liver injury', 'total parenteral nutrition', 'steatohepatitis', and 'vanishing bile duct syndrome'. Potential hepatic graft-versus-host disease cases were then cross-referenced by an exhaustive clinical chart review by an experienced hepatologist (TDS), and the 40 patients were selected based on the integrity of the diagnosis, absence or exclusion of confounders, completeness of information, and extensive clinical, serologic, microbiologic, and radiologic work-up.

All hematoxylin and eosin (H\&E)-stained biopsy slides were blindly evaluated by two hepatopathologists (AES and MIF) for a panel of histologic features. Bile ducts were assessed for the presence or absence of apoptoses, cholangiocyte necrosis, cytoplasmic alteration (eg, eosinophilia, vacuolation), nuclear hyperchromasia, and nuclear disarray (crowding, loss of polarity), each expressed as a percent of bile ducts involved. Cholangiocyte apoptoses were defined as apoptotic bodies-condensed cells with karyorrhectic debris and often surrounded by a white space or halo-within the confines of the bile duct basement membrane. Cholangiocyte necrosis was defined as cholangiocyte nuclear loss, resulting in conspicuous gaps between nuclei. In addition, bile duct damage was scored from 0 to 3 $(0=$ none, $1=$ single cholangiocyte necrosis or apoptosis, 2 = confluent cholangiocyte necrosis, $3=$ bile duct loss), reflecting the average appearance of the portal tracts. Bile duct loss was assessed quantitatively, and expressed as a percent. Twenty-five percent of cases, including both controls and hepatic graft-versus-host disease, had cytokeratin 7 (CK7) performed as part of the original work-up; digitized slides (Scanscope, Aperio) were available on $76 \%$ and bile duct loss was assessed quantitatively. Acidophilic bodies were enumerated per 10 consecutive high power fields (HPF), beginning in the area of greatest acidophilic activity. Cholestasis was scored from 0 to 3 ( $0=$ none, $1=$ zone 3 confined, $2=$ involving zones 2 and 3 , and $3=$ panzonal or bile infarct), based on the most affected area, and location (canalicular, hepatocellular) was noted. Ductular reaction was scored from 0 to $3(0=$ none, $1=$ isolated, 2 =extending beyond two hepatocyte plates, $3=$ extensive), based on the average appearance of the portal tracts. Intraepithelial lymphocytes were scored $0-3$ ( $0=$ none, $1=1,2=2-3,3>3)$, based on the average number of lymphocytes within bile duct epithelium in the specimen. Furthermore, one author (AES), blinded to diagnosis, used digitized slides (Scanscope, Aperio), which were available on $75 \%$ of the cases, to enumerate bile duct cholangiocytes and intraepithelial lymphocytes. A percentage of intraepithelial lymphocytes per cholangiocytes was generated. Portal and lobular inflammation were scored using the Sheuer system. ${ }^{22}$ Presence and degree of portal eosinophils was recorded. The presence or absence of portal vein endotheliitis and hepatic venulitis were also noted.

Hepatic graft-versus-host disease cases were further stratified by clinical definitions of acute $(<100$ days post transplant) and chronic ( $\geq 100$ days post transplant), and into cholestatic and hepatitic forms, where the hepatitic form was defined as hepatic graft-versus-host disease with aspartate 
aminotransferase and/or alanine aminotransferase at least $10 \times$ the normal value. ${ }^{23}$

After statistically significant variables were identified, defined as $P<0.05$, these histologic features were combined into an algorithm, with the goal of creating a simple histologic formula while preserving diagnostic accuracy. After the final algorithm was selected, etiology of control cases was unmasked, such that false positives could be identified.

Statistical analysis was completed using AnalyseIt version 3.9 for Excel. Comparison of histologic factors in hepatic graft-versus-host disease versus controls, and in acute versus chronic hepatic graftversus-host disease, was completed using the Student $t$-test. Sensitivity, specificity, and accuracy were calculated using standard equations.

\section{Results}

Patient and biopsy characteristics of hepatic graftversus-host disease and control groups are shown in Table 1.

Hepatic graft-versus-host disease cases, when compared with the general control group, had statistically greater bile duct damage $(P<0.0001)$, bile duct loss $(P=0.0002)$, cholestasis $(P<0.0001)$, acidophilic bodies $(P=0.0006)$, and statistically less ductular reaction $(P<0.0001)$ (Table 2). Degree of bile duct loss was not significantly different when comparing H\&E and CK7 quantification $(P=0.17)$. Canalicular and hepatocellular cholestasis occurred together, and both were more frequent in hepatic graft-versus-host disease compared with controls $(P<0.0001, P<0.0001)$. There was a trend toward more hepatic venulitis in the control population $(P=0.0617)$. Other histologic parameters were not significantly different between the groups, including intraepithelial lymphocytes evaluated by two methods, even when normal controls were excluded ( $P=0.7854$ by score, $P=0.9545$ by enumeration). There was a statistically significant association between degree of portal inflammation and presence of portal vein endotheliitis $(P<0.0001)$. The spectrum of bile duct damage in hepatic graft-versus-host disease is shown in Figure 1. We found that all studied features of bile duct injury were significantly positively correlated and seen more frequently in hepatic graft-versus-host disease compared with controls (data not shown). References to 'bile duct damage' in the results and discussion are based on the bile duct damage score described in the methods. Lobular alterations in hepatic graft-versus-host disease are shown in Figure 2

When hepatic graft-versus-host disease cases were compared with a drug-induced liver injury-only control group, the same features maintained significance, whereas hepatic venulitis was seen significantly more often in the control group (Table 3).
When hepatic graft-versus-host disease cases were compared with a cholestatic drug-induced liver injury control group, bile duct loss, bile duct damage, acidophilic bodies/10 HPF, and ductular reaction retained significance, but cholestasis became non-significant; however, hepatic venulitis was significantly higher in the control group (Table 3).

When hepatic graft-versus-host disease cases were compared with a vanishing bile duct syndrome pattern of drug-induced liver injury control group, absence of ductular reaction was more often seen in hepatic graft-versus-host disease cases, and there was less bile duct loss (Table 3). A trend toward more hepatic venulitis in control cases was noted.

No histologic differences were found in comparing the acute $(<100$ days post transplant) to chronic ( $\geq 100$ days post transplant) hepatic graft-versus-host disease groups.

Twenty-eight percent of the hepatic graft-versushost disease cases met the definition of hepatitic subtype, with a mean alanine aminotransferase of 602 U/l (range 318-1134) and aspartate aminotransferase of 376 U/l (range 110-813), compared with the cholestatic subgroup, which had means of $139 \mathrm{U} / \mathrm{l}$ (range 46-270) and 85 U/l (range 17-253), respectively. Despite this hepatitic clinical presentation, there was no significant difference in any of the histologic features examined between the hepatitic and cholestatic hepatic graft-versus-host disease groups (Table 4).

Using an abridged scoring system (Table 5) based on the significant histologic findings, we created a simple histologic algorithm for the diagnosis of hepatic graft-versus-host disease with a three-tiered scoring system (Figure 3). Many iterations of the algorithm were evaluated, each using the significant histologic features revealed by data analysis. Weight was placed on bile duct damage and loss. As a simpler surrogate for all features of bile duct damage, we elected to use our bile duct damage score, based on cholangiocyte apoptoses and necrosis and bile duct loss. This score significantly positively correlated with other studied features of bile duct damage, and all cases which had other features of bile duct damage also had apoptoses and/or necrosis. We suspect that assessment apoptoses and necrosis may be less subject to inter-observer variability than other factors; further study is needed. With the goal of making the algorithm as user-friendly as possible, the analysis of other histologic features was streamlined, requiring 'yes/no' responses. The final proposed algorithm is simple, but provides a high accuracy in diagnosis of hepatic graft-versus-host disease and in distinction from the control groups.

When applied to the hepatic graft-versus-host disease and control groups, this algorithm had a sensitivity of $93 \%$, specificity of $93 \%$, accuracy of $92 \%$, negative predictive value of $93 \%$, and positive predictive value of $90 \%$. The most common cause of false positive cases was vanishing bile duct 
Table 1 Patient and liver biopsy characteristics

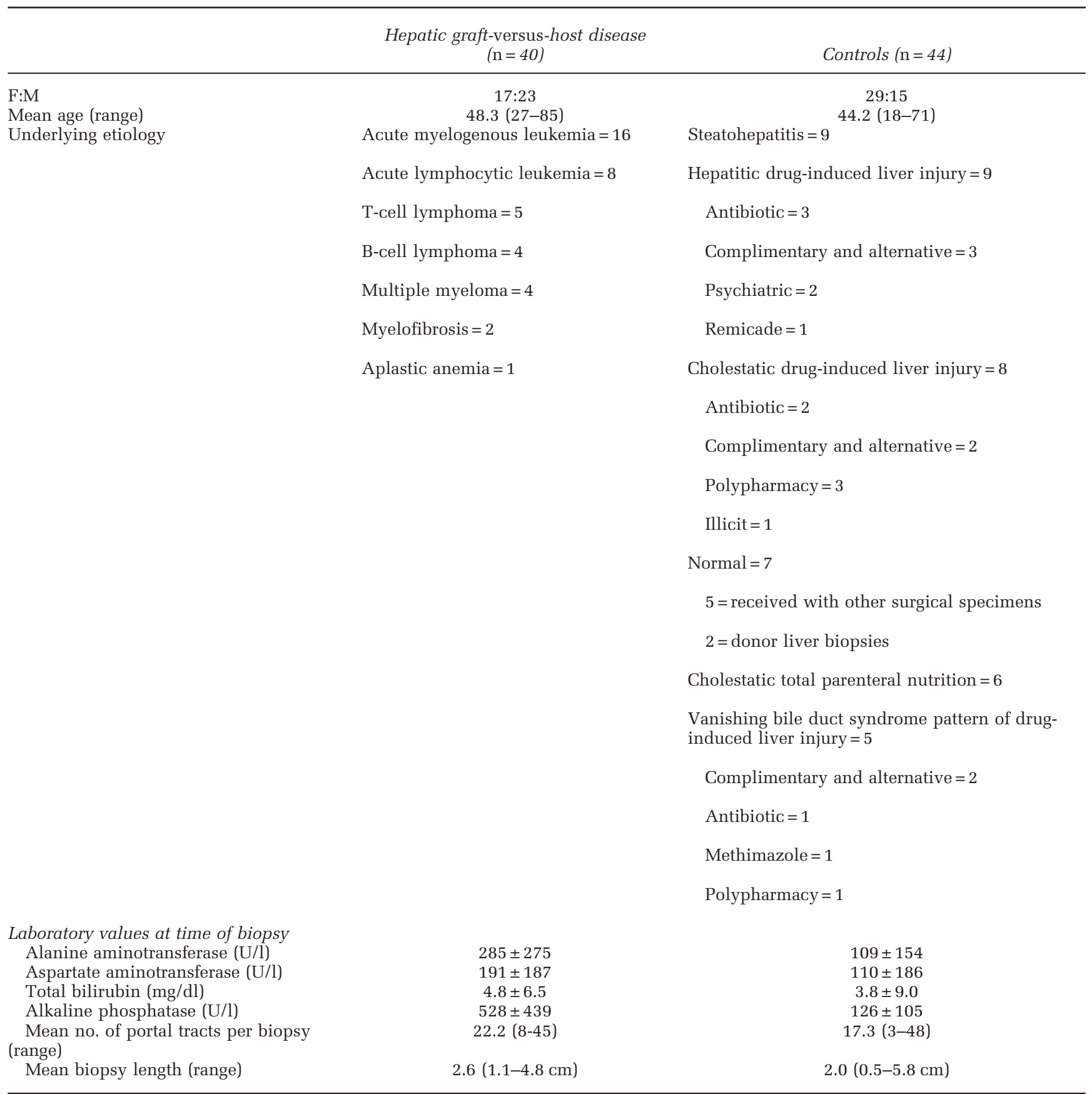

syndrome pattern of drug-induced liver injury. There were four false positives (three vanishing bile duct syndrome, one total parenteral nutrition) and three false negatives. Twenty-five percent of cases stratified to the 'probable' category.

\section{Discussion}

Hepatic graft-versus-host disease is a challenging clinical and pathologic diagnosis. In this study, we have identified histologic features that are significantly different in hepatic graft-versus-host disease compared with controls: greater bile duct loss, bile duct damage, cholestasis, acidophilic bodies, and less ductular reaction. This study is unique in that the control group includes the main clinical and pathologic differential diagnoses of hepatic graftversus-host disease, including cholestatic total parenteral nutrition-related injury, cholestatic druginduced liver injury, and vanishing bile duct syndrome pattern of drug-induced liver injury. In 
Table 2 Histologic features of hepatic graft-versus-host disease cases and controls

\begin{tabular}{|c|c|c|c|}
\hline Features & Hepatic graft-versus-host disease $(\mathrm{n}=40)$ & Controls $(\mathrm{n}=44)$ & P-value \\
\hline Bile duct damage & $2.17 \pm 0.74$ & $0.82 \pm 0.99$ & $<0.0001$ \\
\hline Bile duct loss & $24.31 \pm 15.63$ & $10.77 \pm 16.12$ & 0.0002 \\
\hline Cholestasis & $1.38 \pm 1.21$ & $0.41 \pm 0.76$ & $<0.0001$ \\
\hline Acidophilic bodies/10 HPF & $1.42 \pm 1.44$ & $0.44 \pm 0.85$ & 0.0006 \\
\hline Ductular reaction & $0.32 \pm 0.47$ & $1.00 \pm 0.84$ & $<0.0001$ \\
\hline Intraepithelial lymphocytes & $0.41 \pm 0.50$ & $0.36 \pm 0.53$ & 0.7854 \\
\hline Portal inflammation & $0.76 \pm 0.99$ & $0.93 \pm 0.73$ & 0.3530 \\
\hline Lobular inflammation & $0.88 \pm 0.78$ & $0.93 \pm 0.76$ & 0.7484 \\
\hline Portal vein endotheliitis & $0.22 \pm 0.52$ & $0.34 \pm 0.68$ & 0.3621 \\
\hline Hepatic venulitis & $0.02 \pm 0.16$ & $0.14 \pm 0.35$ & 0.0617 \\
\hline
\end{tabular}

$\mathrm{HPF}=$ high power fields
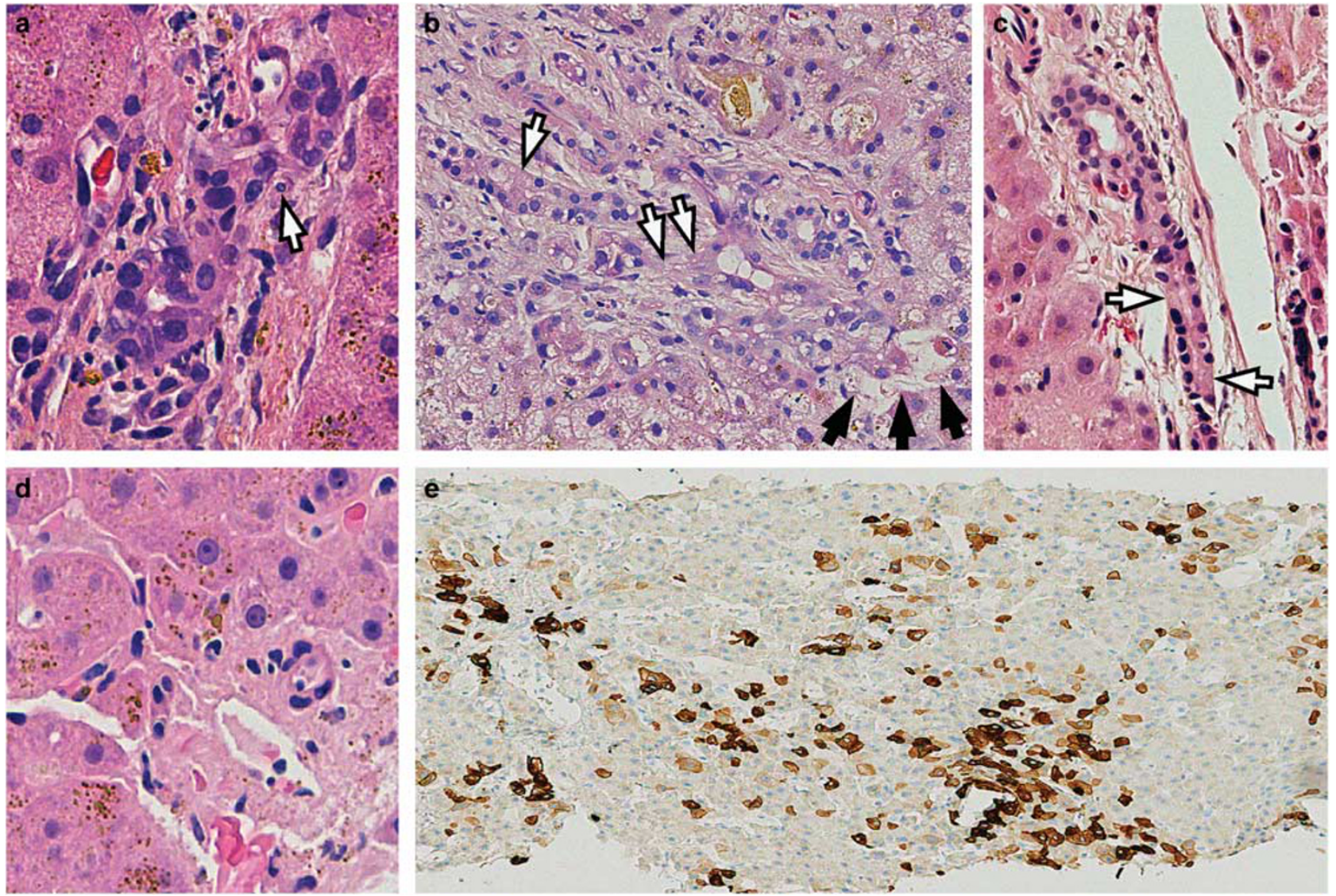

Figure 1 Spectrum of bile duct damage in hepatic graft-versus-host disease. (a) Cholangiocytes showing eosinophilic cytoplasm and variable nuclear hyperchromasia and crowding. An apoptotic body is present (arrow), $40 \times$. (b) Cholangiocytes showing eosinophilic, vacuolated cytoplasm, loss of nuclear polarity, and regions of confluent necrosis with nuclear loss (white arrows). Note the adjacent marked cholestasis and bile infarct (black arrows), $20 \times$. (c) Bile duct with areas of confluent cholangiocyte necrosis (arrows). There is minimal to no ductular reaction, $20 \times$. (d) Portal tract lacking bile ducts, $40 \times$. (e) CK7 immunohistochemistry highlighting intermediate hepatobiliary cells in areas of bile duct loss, $10 \times$.

contrast to many consensus documents and studies, our findings did not show that hepatic graft-versushost disease is enriched for intraepithelial lymphocytes nor portal inflammation; however, it does confirm the robustness of bile duct damage, bile duct loss, cholestasis, and acidophilic bodies in this diagnosis. . $^{9,10,15,16,18,21}$ Differences may be owing to controls. In addition, our results emphasize importance of the absence of ductular reaction and hepatic venulitis in hepatic graft-versus-host disease.

Using our data, we have developed a highly accurate and easy-to-use histologic algorithm, the first of its kind for hepatic graft-versus-host disease. This algorithm may be superior to previously published scoring systems, which predict probability of hepatic graft-versus-host disease; however, further 

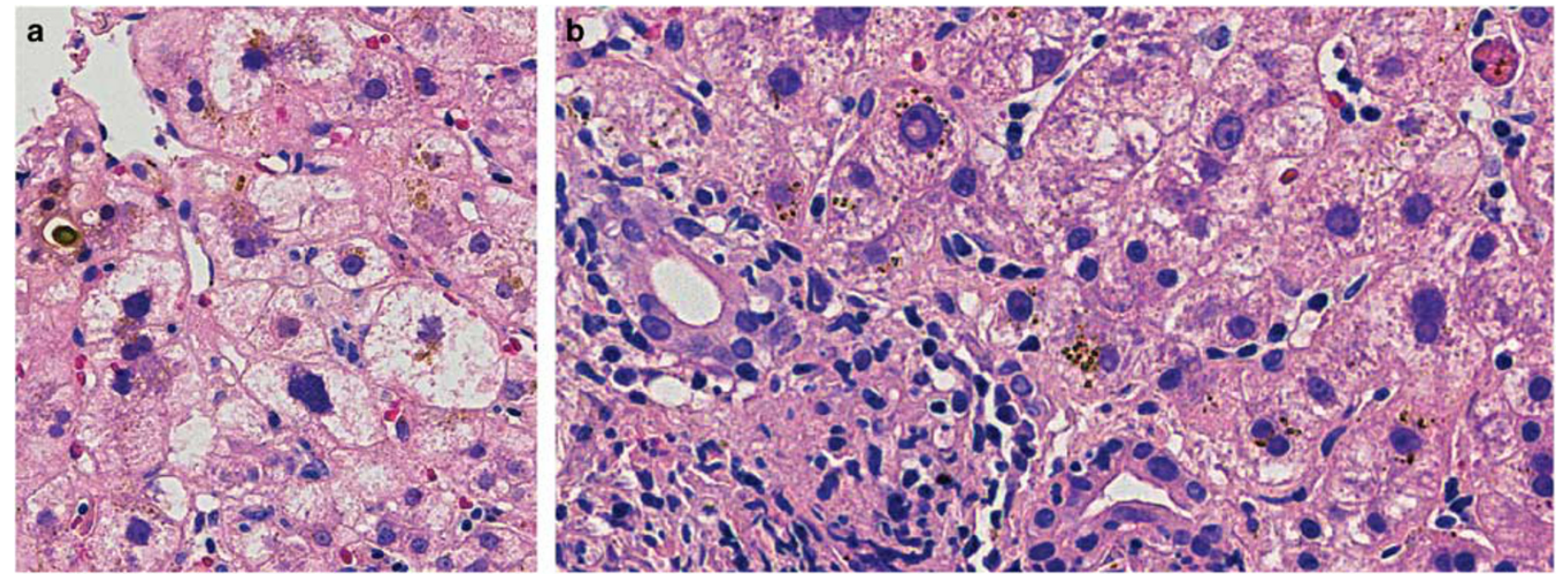

Figure 2 Lobular changes in hepatic graft-versus-host disease. (a) Hepatocellular and canalicular cholestasis, with hepatocyte feathery degeneration, $40 \times$ (b) An acidophilic body is present (top right), along with damaged bile ducts (bottom left), $40 \times$.

studies comparing these systems on the same study group are needed. ${ }^{6,7}$ The algorithm is weighted, with bile duct damage and duct loss accounting for a large number of points; these features appear most important overall in separating hepatic graft-versushost disease from all controls. Additional support points may be gained from other categories, with hepatic venulitis and absence of ductular reaction particularly helpful in comparison with druginduced liver injury controls. Further studies, including prospective testing of this algorithm, and analysis of inter-observer variability in its application, are underway.

The current study emphasizes the importance of liver biopsy in this clinical scenario. Although there are risks associated with liver biopsy, these are cases in which clinical presentation, liver chemistry tests, and current scoring systems are unable to reliably discern the diagnosis of hepatic graft-versus-host disease, making histologic exam necessary. Although biopsy is more invasive than liver chemistry tests used in such scoring systems, the high accuracy of the diagnostic histologic algorithm should be weighed against the risks of biopsy. In addition, other investigators have demonstrated the usefulness of histologic features, including lobular inflammation and hepatocyte ballooning degeneration, on outcome in patients with confirmed hepatic graftversus-host disease. ${ }^{13}$

Drug-induced liver injury is one of the most common clinical differential diagnoses for hepatic graft-versus-host disease. To our knowledge, this is the first study including drug-induced liver injury, and subdivided drug-induced liver injury patterns, within the control group. Our results show that, compared with drug-induced liver injury-only controls, the significant histologic features, namely bile duct loss, bile duct damage, cholestasis, acidophilic bodies, and absence of ductular reaction, are maintained. In addition, we report that hepatic venulitis is a feature in support of drug-induced liver injury, rather than hepatic graft-versus-host disease, and may be used to help differentiate the two. We hypothesize that hepatic venulitis in drug-induced liver injury cases may be related to drug-induced endothelial injury. The presence of hepatic, rather than portal, venulitis could be related to the release of drug metabolites into the sinusoids and hepatic venous drainage, resulting in elevated local concentrations, and/or higher susceptibility to injury owing to the relatively low oxygen environment. The scenario of concurrent hepatic graft-versus-host disease and drug-induced liver injury is possible, but not present in our study population and, thus, not addressed here.

When hepatic graft-versus-host disease cases were compared with a cholestatic drug-induced liver injury control subgroup, presence of cholestasis no longer was significant. However, hepatic graft-versus-host disease cases still showed significantly greater bile duct loss, bile duct damage, and acidophilic bodies, with significantly less ductular reaction and hepatic venulitis. These features can further assist the pathologist in this difficult differential diagnosis.

The most challenging differential is distinguishing hepatic graft-versus-host disease from vanishing bile duct syndrome pattern of drug-induced liver injury, and indeed it was the most common cause of falsepositive results in our algorithm. When we compared our hepatic graft-versus-host disease cases to a vanishing bile duct syndrome pattern of druginduced liver injury control subgroup, we found that hepatic graft-versus-host disease cases had significantly less bile duct loss and less ductular reaction. There was also a trend toward more hepatic venulitis in the vanishing bile duct syndrome pattern of drug-induced liver injury cohort. Thus, in this scenario, the presence of ductular reaction is crucial. It is our own personal observation that vanishing bile 
duct syndrome pattern of drug-induced liver injury biopsies tend to show a mixture of completely uninvolved portal tracts with affected ones, compared with hepatic graft-versus-host disease which shows diffuse involvement of portal tracts. Further study with a larger cohort of vanishing bile duct syndrome cases is warranted.

Hepatitic hepatic graft-versus-host disease, which has been defined by elevations of alanine and/or aspartate aminotransferase $>10 \times$ normal, represents $29-55 \%$ of hepatic graft-versus-host disease. ${ }^{23-25}$ Our data show that despite significantly elevated hepatitic liver chemistry tests, the histology of such cases was identical to those with a cholestatic presentation. Although none of our cases had the histology of severe acute hepatitis, reports of hepatic graft-versus-host disease resembling acute hepatitis, with lobular disarray and even bridging necrosis, exist. ${ }^{26-29}$ It is unclear if these cases truly represent hepatic graft-versus-host disease, another process, or concurrent diagnoses; further study, and exclusion of other causes, such as drug-induced liver injury and viral infection, including Hepatitis E Virus, is needed. ${ }^{30}$ Hepatic graft-versus-host disease may also be associated with de novo liver diseases. Beleoken et al. ${ }^{31}$ noted several hepatic graft-versushost disease patients who went on to develop an autoimmune hepatitis presentation after tapering or cessation of immunosuppression, and suggest that hepatic graft-versus-host disease may be a factor in the pathogenesis of de novo autoimmune liver disease. A primary biliary cholangitis-like picture has been similarly noted in murine models. ${ }^{32-34}$ For such cases, an immune-reconstitution syndrome should also be considered.

The strengths of the current study include the large cohort of clinically confirmed hepatic graftversus-host disease patients with available liver biopsy tissue and control group, which includes the main differential considerations in this clinical and pathologic setting. This is the first study, to our knowledge, to incorporate drug-induced liver injury as part of the control group and have a subgroup analysis, and the first to compare histologic features of hepatic graft-versus-host disease with clinical hepatitic versus cholestatic presentation. The algorithm proposed demonstrates diagnostic reliability and may be superior to other available tools.

In practice, assessment may be limited by the adequacy of the liver biopsy. This may be affected by the type of biopsy; for example, transjugular liver biopsies, which most of this patient population may receive, may be of smaller gauge and contain less portal tracts when compared with a percutaneous sample. Bile duct loss may be challenging to recognize on H\&E-stained sections. Ancillary studies, such as CK7 immunohistochemistry, may be useful. CK7 will highlight preserved bile ducts and ductular reaction. In addition, when a duct is truly lost, periportal hepatocytes become metaplastic, referred to as 'intermediate hepatobiliary cells', and 
Table 4 Histologic and biochemical features of hepatitic versus cholestatic hepatic graft-versus-host disease cases

\begin{tabular}{|c|c|c|c|}
\hline Features & $\begin{array}{l}\text { Hepatitic hepatic graft-versus-host disease } \\
\qquad(\mathrm{n}=11)\end{array}$ & $\begin{array}{l}\text { Cholestatic hepatic graft-versus-host disease } \\
\qquad(\mathrm{n}=29)\end{array}$ & $\mathrm{P}$-value \\
\hline Bile duct damage & $2.27 \pm 0.21$ & $2.17 \pm 0.15$ & 0.7010 \\
\hline Bile duct loss & $25.82 \pm 4.95$ & $24.94 \pm 3.43$ & 0.8852 \\
\hline Cholestasis & $1.36 \pm 0.38$ & $1.57 \pm 0.26$ & 0.6636 \\
\hline Acidophilic bodies/10 HPF & $1.82 \pm 0.43$ & $1.41 \pm 0.30$ & 0.4428 \\
\hline Ductular reaction & $0.27 \pm 0.24$ & $0.30 \pm 0.10$ & 0.8554 \\
\hline Intraepithelial lymphocytes & $0.36 \pm 0.15$ & $0.39 \pm 0.10$ & 0.8811 \\
\hline Portal inflammation & $0.64 \pm 1.31$ & $1.00 \pm 0.22$ & 0.3469 \\
\hline Lobular inflammation & $1.18 \pm 0.23$ & $0.87 \pm 0.16$ & 0.2677 \\
\hline Portal vein endotheliitis & $0.18 \pm 0.18$ & $0.39 \pm 0.12$ & 0.3395 \\
\hline Hepatic venulitis & $0.09 \pm 0.07$ & $0.04 \pm 0.05$ & 0.5958 \\
\hline Alanine aminotransferase (U/l) & $601.73 \pm 52.22$ & $138.52 \pm 36.11$ & $<0.0001$ \\
\hline Aspartate aminotransferase (U/l) & $376.36 \pm 39.77$ & $84.70 \pm 27.50$ & $<0.0001$ \\
\hline Total bilirubin (mg/dl) & $4.72 \pm 2.02$ & $4.76 \pm 1.39$ & 0.9876 \\
\hline Alkaline phosphatase (U/l) & $507.64 \pm 136.26$ & $532.13 \pm 94.23$ & 0.8834 \\
\hline
\end{tabular}

$\mathrm{HPF}=$ high power fields.

Table 5 Hepatic graft-versus-host disease probability score

\begin{tabular}{|c|c|c|c|c|c|}
\hline Feature & Score & 0 & 1 & 2 & 3 \\
\hline Bile duct loss (\% of portal tracts) & & $0-9$ & $10-24$ & $25-49$ & $\geq 50$ \\
\hline $\begin{array}{l}\text { Bile duct damage (dominant bile } \\
\text { duct appearance in biopsy) }\end{array}$ & & None & $\begin{array}{l}\text { Bile ducts with single } \\
\text { cholangiocyte necrosis or } \\
\text { apoptosis }\end{array}$ & $\begin{array}{l}\text { Bile ducts with confluent } \\
\text { cholangiocyte necrosis or apoptoses }\end{array}$ & $\begin{array}{l}\text { Bile duct } \\
\text { loss }\end{array}$ \\
\hline Acidophilic bodies per $10 \mathrm{HPF}^{\mathrm{a}}$ & & 0 & $\geq 1$ & NA & NA \\
\hline Cholestasis, any degree & & Absent & Present & NA & NA \\
\hline Ductular reaction, any degree & & Absent & Present & NA & NA \\
\hline
\end{tabular}

$\mathrm{HPF}=$ high power field.

${ }^{\mathrm{a}}$ Area of highest acidophilic activity chosen as first HPF.

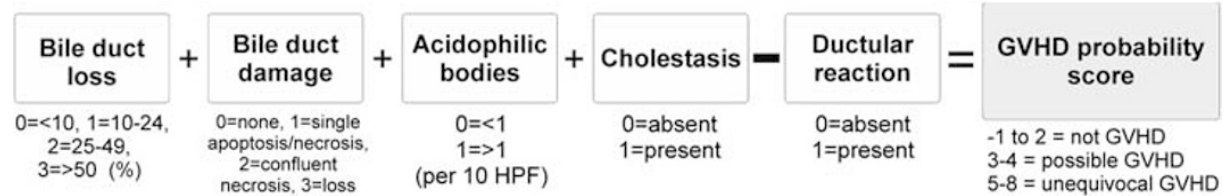

Figure 3 Novel histologic algorithm to predict likelihood of hepatic graft-versus-host disease.

show expression of cytoplasmic CK7. A rhodanine stain may highlight periportal copper accumulation associated with cholate stasis of chronic duct loss.

As part of prospective interrogation of the algorithm, one author (AES) has received two biopsies from patients post stem cell transplantation, and evaluated the pathology, including application of the algorithm, prior to reviewing any further history or clinical impression. The first biopsy showed diffuse bile duct damage, with areas of confluent cholangiocyte necrosis, $20 \%$ bile duct loss, scattered acidophilic bodies, mild cholestasis, and absence of ductular reaction and endotheliitis. It categorized into unequivocal hepatic graft-versus-host disease (score 5) per the algorithm. The patient was a 51year-old male with history of chronic myelogenous leukemia and diffuse large B-cell lymphoma who underwent stem cell transplantation one month prior to biopsy. He was not able to tolerate the full regimen of immunosuppression after transplantation, and had received numerous antibiotics and antifungals in the prior month. Biopsy was performed for elevated liver transaminase activity levels and bilirubin, with a clinical differential of druginduced liver injury, infection, and hepatic graftversus-host disease. After the liver biopsy, the patient was placed on additional immunosuppression and fluconazole was discontinued. Liver enzymes and function improved in the following days, but did not normalize. The patient died of multiorgan failure 5 days after liver biopsy. Owing to the short survival post biopsy, and concurrent cessation of fluconazole and increase in immunosuppression, it is difficult to draw conclusions in this case. The second biopsy showed diffuse bile duct damage, $25 \%$ bile duct loss, marked cholestasis with bile infarcts, presence of acidophilic bodies, and absence of endotheliitis and ductular reaction. Per the algorithm, it corresponded to unequivocal hepatic graft-versus-host disease (score 6). The patient was a 53-year-old man who underwent stem cell transplantation for refractory follicular lymphoma 
four years prior, had a history of intestinal graftversus-host disease treated two years prior, and had recent transformation to diffuse large B-cell lymphoma, at which time immunosuppressants were stopped. He developed new bilirubin and transaminase activity level elevation, and the clinical differential included hepatic graft-versus-host disease, infection (the patient had a history of Cytomegalovirus and shingles), and involvement by lymphoma. A skin biopsy was done at the same time as the liver biopsy, and later reported as compatible with graft-versus-host disease. After the liver biopsy, the patient was started on cyclosporine and prednisone, and liver function and transaminase activity levels improved. The patient died of lymphoma seven months later. In this case, the course, response to treatment, and lack of confounding treatment alterations support the diagnosis of hepatic graft-versus-host disease and accuracy of the algorithm. Prospective analysis of additional cases is required.

In conclusion, we have combined several specific significant histologic parameters into a simple, accurate hepatic graft-versus-host disease probability score and three-tiered scoring system, allowing for incorporation into clinical practice. This algorithm is able to accurately distinguish between mimickers and hepatic graft-versus-host disease, whether having a hepatitic or cholestatic presentation. In comparison with currently used scoring systems and consensus documents, which guide clinical decisions, our algorithm appears to provide improved sensitivity, specificity, and accuracy.

\section{Disclosure/conflict of interest}

The authors declare no conflict of interest.

\section{References}

1 Kim BK, Chung KW, Sun HS, et al. Liver disease during the first post-transplant year in bone marrow transplantation recipients: retrospective study. Bone Marrow Transplant 2000;26:193-197.

2 Miglio F, Mazzeo V, Baraldini M, et al. Graft versus host of the liver: clinical, morphological and immunological aspects. Prog Clin Biol Res 1989;309:239-247.

3 Kambham N, Higgins JP, Sundram U, et al. Hematopoietic stem cell transplantation: graft versus host disease and pathology of gastrointestinal tract, liver, and lung. Adv Anat Pathol 2014;21:301-320.

4 Jagasia MH, Greinix HT, Arora M, et al. National Institutes of Health Consensus Development Project on Criteria for Clinical Trials in Chronic Graft-versus-Host Disease: I. The 2014 Diagnosis and Staging Working Group report. Biol Blood Marrow Transplant 2015;21: 389-401.

5 Shulman HM, Cardona DM, Greenson JK, et al. NIH Consensus development project on criteria for clinical trials in chronic graft-versus-host disease: II. The 2014
Pathology Working Group Report. Biol Blood Marrow Transplant 2015;21:589-603.

6 Han MAT, Samala N, Rizvi BS, et al. Liver enzymes elevation in the setting of chronic graft-versus-hostdisease is a non specific marker of inflammation that does not accurately predict disease related hepatic injury. Hepatology 2015;62:1251A.

7 Han MAT SN, Rizvi BS, Etzion O, et al. Abnormal liver tests are inaccurate for diagnosis of chronic hepatic graft versus host disease in critically ill patients. Hepatology 2016;64:182A.

8 Andersen CB, Horn T, Sehested M, et al. Graft-versushost disease: liver morphology and pheno/genotypes of inflammatory cells and target cells in sex-mismatched allogeneic bone marrow transplant patients. Transplant Proc 1993;25:1250-1254.

9 Berman MD, Rabin L, O'Donnell J, et al. The liver in long-term survivors of marrow transplant-chronic graft-versus-host disease. J Clin Gastroenterol 1980;2: 53-63.

10 Bernuau D, Gisselbrecht C, Devergie A, et al. Histological and ultrastructural appearance of the liver during graft-versus-host disease complicating bone marrow transplantation. Transplantation 1980;29:236-244.

11 Beschorner WE, Pino J, Boitnott JK, et al. Pathology of the liver with bone marrow transplantation. Effects of busulfan, carmustine, acute graft-versus-host disease, and cytomegalovirus infection. Am J Pathol 1980;99: 369-386.

12 Chiba T, Yokosuka O, Goto S, et al. Clinicopathological features in patients with hepatic graft-versus-host disease. Hepatogastroenterology 2005;52:1849-1853.

13 Duarte RF, Delgado J, Shaw BE, et al. Histologic features of the liver biopsy predict the clinical outcome for patients with graft-versus-host disease of the liver. Biol Blood Marrow Transplant 2005;11:805-813.

14 Nonomura A, Kono N, Mizukzmi Y, et al. Histological changes in the liver in experimental graft-versus-host disease across minor histocompatibility barriers. VI. A light and electron microscopic study of the periportal changes. Liver 1991;11:278-286.

15 Nonomura A, Kono N, Yoshida K, et al. Histological changes of the liver in experimental graft-versus-host disease across minor histocompatibility barriers. V. A light and electron microscopic study of the intralobular changes. Liver 1991;11:149-157.

16 Quaglia A, Duarte R, Patch D, et al. Histopathology of graft versus host disease of the liver. Histopathology 2007;50:727-738.

17 Glucksberg H, Storb R, Fefer A, et al. Clinical manifestations of graft-versus-host disease in human recipients of marrow from HL-A-matched sibling donors. Transplantation 1974;18:295-304.

18 Shulman HM, Sharma P, Amos D, et al. A coded histologic study of hepatic graft-versus-host disease after human bone marrow transplantation. Hepatology 1988;8:463-470.

19 Snover DC, Weisdorf SA, Ramsay NK, et al. Hepatic graft versus host disease: a study of the predictive value of liver biopsy in diagnosis. Hepatology 1984;4: 123-130.

20 Lerner KG, Kao GF, Storb R, et al. Histopathology of graft-vs.-host reaction (GvHR) in human recipients of marrow from HL-A-matched sibling donors. Transplant Proc 1974;6:367-371.

21 Stift J, Baba HA, Huber E, et al. Consensus on the histopathological evaluation of liver biopsies from 
patients following allogeneic hematopoietic cell transplantation. Virchows Arch 2014;464:175-190.

22 Desmet VJ, Gerber M, Hoofnagle JH, et al. Classification of chronic hepatitis: diagnosis, grading and staging. Hepatology 1994;19:1513-1520.

23 Ma SY, Au WY, Lie AK, et al. Liver graft-versus-host disease after donor lymphocyte infusion for relapses of hematologic malignancies post allogeneic hematopoietic stem cell transplantation. Bone Marrow Transplant 2004;34:57-61.

24 Strasser SI, Shulman HM, Flowers ME, et al. Chronic graft-versus-host disease of the liver: presentation as an acute hepatitis. Hepatology 2000;32:1265-1271.

25 Akpek G, Boitnott JK, Lee LA, et al. Hepatitic variant of graft-versus-host disease after donor lymphocyte infusion. Blood 2002;100:3903-3907.

26 Chiba T, Yokosuka O, Kanda T, et al. Hepatic graftversus-host disease resembling acute hepatitis: additional treatment with ursodeoxycholic acid. Liver 2002;22:514-517.

27 Fujii N, Takenaka K, Shinagawa K, et al. Hepatic graftversus-host disease presenting as an acute hepatitis after allogeneic peripheral blood stem cell transplantation. Bone Marrow Transplant 2001;27:1007-1010.

28 Maeng H, Lee JH, Cheong JW, et al. Chronic graftversus-host disease of the liver presenting as an acute hepatitis following nonmyeloablative hematopoietic stem cell transplantation. Int J Hematol 2004;79: 501-504.
29 Mori T, Yokoyama A, Aisa Y, et al. A flare-up of chronic graft-versus-host disease of the liver that mimics acute hepatitis in a recipient of an allogeneic peripheral blood stem cell transplant. Int J Hematol 2005;81:174-175.

30 Davern TJ, Chalasani N, Fontana RJ, et al. Acute hepatitis $\mathrm{E}$ infection accounts for some cases of suspected drug-induced liver injury. Gastroenterology 2011;141:1665-1672.

31 Beleoken E, Sobesky R, Le Caer JP, et al. Immunoproteomic analysis of potentially severe non-graft-versushost disease hepatitis after allogenic bone marrow transplantation. Hepatology 2013;57:689-699.

32 Suzuki K, Narita T, Yui R, et al. Mechanism of the induction of autoimmune disease by graft-versus-host reaction. Role of CD8+ cells in the development of hepatic and ductal lesions induced by CD4+ cells in MHC class I plus II-different host. Lab Invest 1994;70: 609-619.

33 Itoh S, Matsuzaki Y, Kimura T, et al. Cytokine profile of liver-infiltrating CD4+ T cells separated from murine primary biliary cirrhosis-like hepatic lesions induced by graft-versus-host reaction. J Gastroenterol Hepatol 2000;15:443-451.

34 Vierling JM, Hreha G, Wang $\mathrm{H}$, et al. The role of biliary epithelial cells in the immunopathogenesis of nonsuppurative destructive cholangitis in murine hepatic graft-versus-host disease. Trans Am Clin Climatol Assoc 2011;122:326-335. 